\title{
Unconventional time-bandwidth performance of resonant cavities with nonreciprocal coupling
}

\author{
Ivan Cardea ${ }^{1}$, Davide Grassani ${ }^{2}$, Jeremy Upham ${ }^{3}$, Sebastian A. Schulz ${ }^{4}$, \\ Kosmas L. Tsakmakidis ${ }^{5}$, Camille-Sophie Brès ${ }^{1 *}$ \\ ${ }^{1}$ Ecole Polytechnique Fédérale de Lausanne (EPFL), Photonic Systems Laboratory \\ (PHOSL), Lausanne CH-1015, Switzerland \\ ${ }^{2}$ Dipartimento di Fisica, Università degli Studi di Pavia, via Bassi 6, 27100 Pavia, Italy \\ ${ }^{3}$ Department of Physics, University of Ottawa, Ottawa, ON, Canada \\ ${ }^{4}$ School of Physics and Astronomy, SUPA, University of St Andrews, St Andrews, KY169SS, \\ $U K$ \\ ${ }^{5}$ Section of Condensed Matter Physics, Department of Physics, National and Kapodistrian \\ University of Athens, Panepistimioupolis, GR - 157 84, Athens, Greece \\ *Correspondence to: camille.bres@epfl.ch
}

The time-bandwidth limit is a mathematical tenet that affects all reciprocal resonators, stating that the product of the spectral bandwidth that can couple into a resonant system and its characteristic energy decay time is always equal to 1 . Here, we develop an analytical and numerical model to show that introducing nonreciprocal coupling to a generalized resonator changes the power balance between the reflected and intra-cavity fields, which consequently overcomes the time-bandwidth limit of the resonant system. By performing a full evaluation of the time-bandwidth product (TBP) of the modeled resonator, we show that it represents a measure of the increased delay imparted to a light wave, with respect to what the bandwidth of the reciprocal resonant structure would allow to the same amount of in-coupled power. No longer restricted to the value 1 , we show that the TBP can instead be used as a figure of merit of the improvement in intra-cavity power enhancement due to the nonreciprocal coupling.

\section{INTRODUCTION}

The capability to slow down or trap light without imposing an excessive distortion to the signal is a key tool of many research areas such as optical communications [1,2], quantum information processing [3], metamaterials [4] and photovoltaics [5-7]. In particular, it is of fundamental importance in all the applications requiring optical signal processing or light storage [8-14]. Thus far, owing to the progress attained in the development of slow light devices, new frontiers of light propagation have been achieved, in which light can be dramatically slowed [15], captured and then released at a later time [16-18] or even stopped all together [19-21]. Generally, what is required from the practical point of view is the ability 
to impart a delay to a signal that is independent of the signal's bandwidth. For instance, in wavelength division multiplexed (WDM) multichannel systems, storage devices and delay lines are used at the receiver end to store high-rate data packets as they are read out at slower rate or for queuing while the transmitter awaits access to the network [22,23].

In the last two decades, different implementations of resonant structures have been explored for the realization of delay lines and storage devices [24-31], since they offer a long effective delay at small footprints. Resonant cavities are also widely used in nonlinear optics applications, such as frequency comb and Kerr soliton generation [32,33], where the intracavity power enhancement helps to reduce the input power required to induce nonlinear effects. As it is well known, reducing the cavity bandwidth is a way to increase the interaction time, as well as the power enhancement factor. However, this leads to a constraint that unavoidably imposes a trade-off between the storage/delay time achievable and the width of the operational spectral bandwidth [34-36]. In mathematical terms, this trade-off is described by the timebandwidth limit, a fundamental rule that arises from Fourier-reciprocity considerations, which dictates that the time-bandwidth product (TPB) must be $\Delta \omega \cdot \tau=1$, with $\Delta \omega$ the system bandwidth and $\tau$ the energy decay time [37-39]. Both high-finesse and losses limit the storage capacity of microresonator-based devices [40]. Precisely the same issue limits slow light devices, which are equivalently limited by large group velocity dispersion and losses $[8,41]$.

Recently, a proposal [42] indicated that breaking Lorenz reciprocity can overcome the timebandwidth limit in a resonant system. Following this idea, we have experimentally demonstrated [43] a fully nonreciprocal cavity with TBP exceeding the 'fundamental' limit by a factor of 30 and limited only by intrinsic losses. We also proposed that the concept of the bandwidth that the resonator can "accept" (the acceptance bandwidth $\Delta \omega_{\mathrm{acc}}$ ) can be different from what is considered the cavity bandwidth $\Delta \omega_{\text {cav }}$, which is simply the inverse of the characteristic decay time $(\tau)$.

Here, we present a generalized theoretical model of a resonant cavity having a nonreciprocal coupling element. We analytically derive the frequency response of the reflected and intra-cavity fields, their associated powers and the TBP of the system as a function of the degree of nonreciprocity, i.e. the difference between the in-coupling and out-coupling energy rate. We numerically validate this model simulating a cavity where nonreciprocity is obtained by breaking its time-invariance [44], as it provides a direct way to implement this device using off-the-shelf telecom components [43]. Our results show that resonant systems with a nonreciprocal coupling can provide a longer delay/storage time and a significant improvement 
of the intra-cavity power enhancement, with respect to their reciprocal counterpart, which is strongly desirable in all the applications that demand high efficiency in nonlinear processes. Moreover, we demonstrate that such improvement of intra-cavity power enhancement is nothing but the TBP of the system, quantitatively linking the degree of nonreciprocity of a resonator to a figure of merit for nonlinear optical processes.

\section{THEORETICAL MODEL}

\section{A. Resonant system with nonreciprocal coupling}

To analyze a resonant system with nonreciprocal coupling, we consider a Gires-Tournois resonator $[45,46]$. The back mirror (M2) is fully reflective, while the front mirror (M1) is characterized by the following generic scattering matrix:

$$
\mathbf{S}_{\mathrm{M} 1}=\left[\begin{array}{ll}
\mathbf{t}_{12} & \mathbf{r}_{21} \\
\mathbf{r}_{12} & \mathbf{t}_{21}
\end{array}\right]
$$

where $\mathbf{t}_{12}\left(\mathbf{t}_{21}\right)$ and $\mathbf{r}_{12}\left(\mathbf{r}_{21}\right)$ are the complex transmission and reflection coefficients respectively of a wave incident from outside (inside) the resonator. A schematic illustration of such resonant system is shown in Fig. 1, where $A, A_{\text {in }}$ and $A_{\mathrm{R}}$ represent the complex amplitudes of the intracavity, the incoming and the (total) reflected wave respectively. In this system the front mirror constitutes the only physical port. Therefore, the total in- and out-coupling radiative energy rates, $\rho_{\mathrm{in}}$ and $\rho_{\text {out }}$, respectively, are given by $\rho_{\mathrm{in}}=\left|\mathbf{t}_{12}\right|^{2} / T_{\mathrm{RT}}$ and $\rho_{\text {out }}=\left|\mathbf{t}_{21}\right|^{2} / T_{\mathrm{RT}}$, with $T_{\mathrm{RT}}$ being the cavity round-trip time, while $\rho_{0}$ is the intrinsic, or non-radiative, energy decay rate. A nonreciprocal coupling implies that $\rho_{\text {in }}$ and $\rho_{\text {out }}$ are different due to a nonreciprocal transmittance of the front mirror $\left(\left|\mathbf{t}_{12}\right|^{2} \neq\left|\mathbf{t}_{21}\right|^{2}\right)$ [43]. Consequently, the difference $\left|\mathbf{t}_{12}\right|^{2}-\left|\mathbf{t}_{21}\right|^{2}$ can be seen as a measure of the degree of nonreciprocity of the system. Note that the analytical model aims at studying the implication of decoupling input and output energy rates in a resonant system irrespective to the mechanism used to induce the nonreciprocal coupling, which, in any case, must ensure the conservation of energy [47-50]. In particular, the model does not presume how the scattering matrix with such characteristics is generated, thus the origin of the nonreciprocity (e.g. external magnetic field bias, temporal variance, nonlinearity, etc.) $[47,51]$ does not have any impact on the TBP and the power balance of the system: as long as the system has such scattering matrix, it can exhibit nonreciprocal coupling. In the following sections, we show that temporal variance is one way to reach this state, but other mechanisms, such as external magnetic field bias or nonlinearity, could also lead to the same outcome. Moreover, although, for the sake of simplicity, we are considering a one-port system, 
the model can be generalized for multi-port systems just by taking into account the in- and outcoupling energy rates of the other ports.

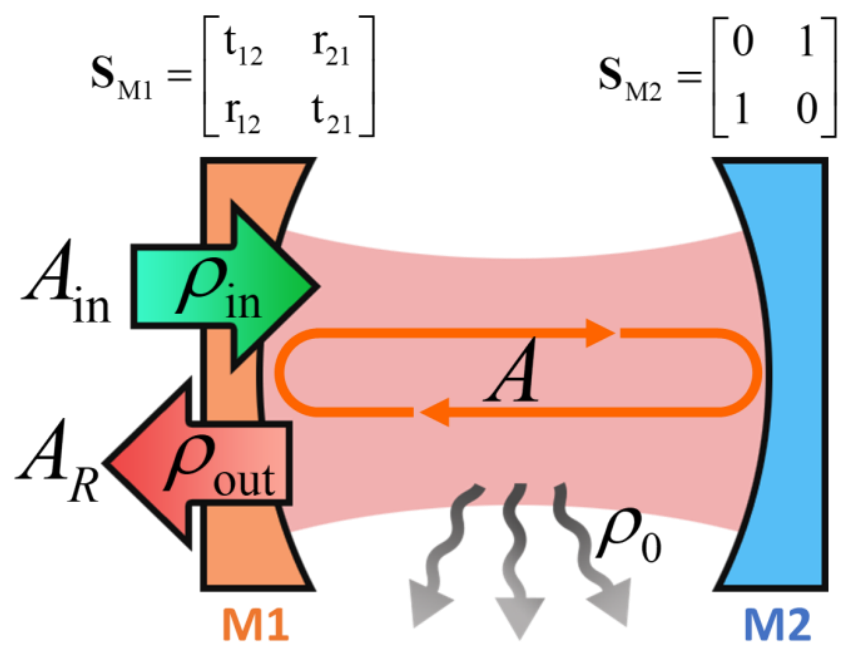

FIG. 1. (a) Layout of a Gires-Tournois resonator with a nonreciprocal front mirror, whose transmission coefficients depend on the direction of wave propagation, and a fully reflective rear mirror.

\section{B. Derivation of the frequency response}

To characterize the frequency response of the system, we use the formalism of the coupling of modes in space also known as power coupling theory (PCT) $[37,46,52]$. While the temporal coupled mode theory (TCMT) [37,53-56] can also be used to describe the spectral distribution of a resonant mode in an optical cavity, even in the context of nonreciprocity $[57,58]$, the equations on which it is based can approximate the spectral response of a resonator only under the assumption of weak coupling. By using the PCT we therefore carry out an analysis unconstrained by coupling strength assumptions and that can also consider multiple resonant mode profiles. Another distinction is that PCT gives the frequency response of the resonator, which has the shape of an Airy function, while the TCMT approximates the resonant system as a Lorentz oscillator characterized by a single longitudinal mode [59].

The spectral distribution of the intra-cavity $(A)$ and reflected $\left(A_{\mathrm{R}}\right)$ fields can be expressed as follows (details in Appendix A):

$$
\begin{gathered}
A(\omega)=\frac{\sqrt{\rho_{\mathrm{in}} T_{\mathrm{RT}}} \sqrt{a_{\mathrm{d}}}}{1-\exp \left[\ln \left(\left|\mathbf{r}_{21}\right|\right)-\left(\rho_{0} / 2+j \Delta \omega\right) T_{\mathrm{RT}}\right]} A_{\mathrm{in}}(\omega) \\
A_{\mathrm{R}}(\omega)=\left[\mathbf{r}_{12}+\frac{\sqrt{\rho_{\mathrm{in}} \rho_{\mathrm{out}}} T_{\mathrm{RT}} a_{\mathrm{d}} \exp \left[-j\left(\Delta \omega T_{\mathrm{RT}}-\phi_{21}^{\mathrm{r}}\right)\right]}{1-\exp \left[\ln \left(\left|\mathbf{r}_{21}\right|\right)-\left(\rho_{0} / 2+j \Delta \omega\right) T_{\mathrm{RT}}\right]}\right] A_{\text {in }}(\omega)
\end{gathered}
$$

where $\phi^{\mathbf{r}_{21}}$ is the phase of $\mathbf{r}_{21}, a_{\mathrm{d}}$ is the field inner circulation factor that accounts for the nonradiative loss of the resonator, $\Delta \omega=\omega-\omega_{0}$ is the frequency detuning from resonance and, the 
field of the incident wave $A_{\text {in }}(\omega)$ is assumed having a flat frequency distribution over one free spectral range $\left(\mathrm{FSR}=1 / T_{\mathrm{RT}}\right)$.

\section{Analysis of the power balance of the resonant system}

To study the effect of nonreciprocity on the power balance of the resonator, we calculate the total reflected and intra-cavity powers encased in one FSR. When normalized to the input power and the FSR, expressed in angular frequency $\left(\Delta \omega_{\mathrm{FSR}}=2 \pi / T_{\mathrm{RT}}\right)$, they are given by the following expressions respectively:

$$
G_{\mathrm{R}}=\frac{1}{\Delta \omega_{\mathrm{FSR}}} \int_{\mathrm{FSR}}\left|\frac{A_{\mathrm{R}}(\omega)}{A_{\mathrm{in}}(\omega)}\right|^{2} d \omega \quad G_{\mathrm{cav}}=\frac{1}{\Delta \omega_{\mathrm{FSR}}} \int_{\mathrm{FSR}}\left|\frac{A(\omega)}{A_{\mathrm{in}}(\omega)}\right|^{2} d \omega
$$

where the argument of the integral of $G_{\mathrm{cav}}$ is nothing else but the intra-cavity power enhancement. Therefore, $G_{\mathrm{cav}}$ represents the total power enhancement attained over one FSR.

First, we study the problem focusing on a purely theoretical analysis, plotting the values of $G_{\text {cav }}$ and $G_{\mathrm{R}}$, as a function of the in- and out-coupling transmittance. Figure 2a shows the case with $a_{\mathrm{d}}{ }^{2}=0.1 \mathrm{~dB}, T_{\mathrm{RT}}=100 \mathrm{~ns}$ and the phases of $\mathbf{r}_{12}$ and $\mathbf{r}_{21}$ both set to 0 . The red lines indicate the states where coupling is reciprocal $\left(\left|\mathbf{t}_{12}\right|^{2}=\left|\mathbf{t}_{21}\right|^{2}\right)$. The maximum value of $G_{\text {cav }}$, occurs when $\left|\mathbf{t}_{12}\right|^{2}=1$ and $\left|\mathbf{t}_{21}\right|^{2}=0$, i.e. when there is total inwards transmission and zero outwards transmission through the front mirror. Conversely, when $\left|\mathbf{t}_{12}\right|^{2}=0$, nothing enters in the resonators and, as expected, $G_{\text {cav }}=0$. More importantly, we note that, owing to the nonreciprocal coupling, the intra-cavity power can be enhanced by more than a factor of 40 with respect to the reciprocal case (red line). The graph of $G_{\mathrm{R}}$ shows an inverse behavior, with a peak value occurring at $\left|\mathbf{t}_{12}\right|^{2}=0$, and a minimum when $\left|\mathbf{t}_{12}\right|^{2}=1$ and $\left|\mathbf{t}_{21}\right|^{2}=0$. The graphs in Figs. $2 \mathrm{~b}$ and $2 \mathrm{c}$ are for 1 and $2 \mathrm{~dB}$ of internal (round-trip) loss, respectively. We can see that $G_{\text {cav }}$ decreases with increasing internal loss for all combinations of $\left|\mathbf{t}_{12}\right|^{2}$ and $\left|\mathbf{t}_{21}\right|^{2}$, while the maximum value of $G_{\mathrm{R}}$ does not change because, in this case, the contribution of the out-coupled power, that is affected by the internal loss, is missing. We also note that $G_{\mathrm{R}}$ exhibits smoother variations as a function of $\left|\mathbf{t}_{21}\right|^{2}$ when the contribution of the internal loss increases. 

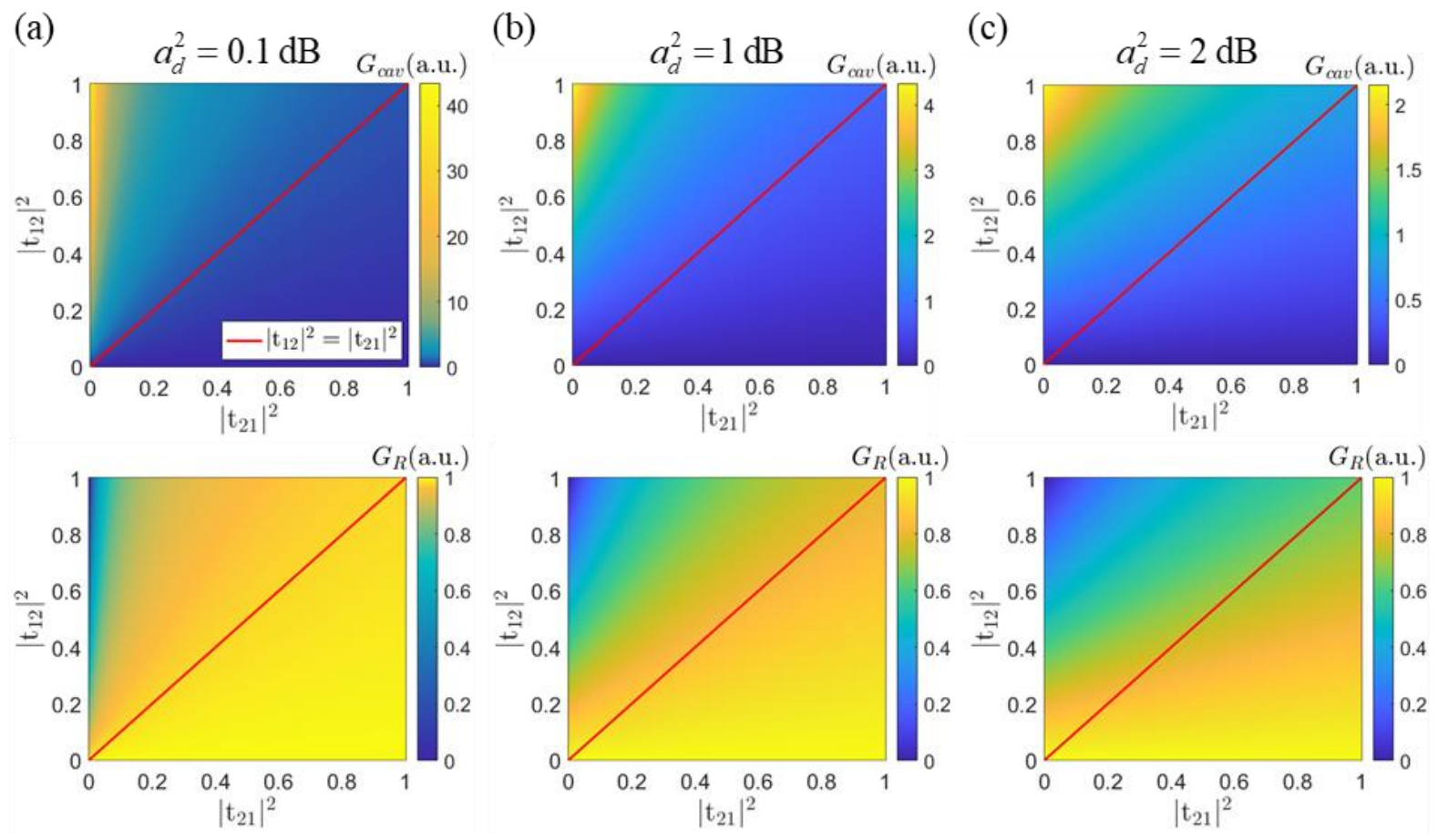

FIG. 2. Graphs in color scale of the total intra-cavity (top row) and reflected (bottom row) power encased in one FSR, normalized to the total input power and $\Delta \omega_{\mathrm{FSR}}$, plotted as a function of the incoupling and out-coupling transmittances. The values are related to a resonator with (a) $0.1 \mathrm{~dB}$, (b) 1 $\mathrm{dB}$ and (c) $2 \mathrm{~dB}$ of internal loss, while the red line indicates the points relative to the reciprocal coupling.

To validate the theoretical analysis, we compare these results with those obtained from simulations based on a full-wave analysis conducted using the software VPIphotonics (https://www.vpiphotonics.com). Although the model does not depend on the mechanism used to induce the nonreciprocal coupling, we implement the Gires-Tournois resonator in the form of a figure- 9 cavity where the nonreciprocal front mirror is simulated by a time-modulated Sagnac interferometer, as it has been proven to be experimentally implemented with standard telecommunication components [43]. Details on the numerical setup used in the simulations can be found in Appendix B. Through this numerical model, we could arbitrarily and independently vary the in- and out-coupling transmission coefficients of the front mirror. However, for the sake of clarity, we analyze the system for two different cases. As the degree of nonreciprocity is equivalent to the minimum distance from the line of reciprocity for any point in the parameter space considered in Fig. 2, in case (A) we evaluate the spectral response of the system for different degrees of nonreciprocity by considering points meeting the conditions $\left|\mathbf{t}_{12}\right|^{2}+\left|\mathbf{t}_{21}\right|^{2}=1$ and $\left|\mathbf{t}_{12}\right|^{2} \geq\left|\mathbf{t}_{21}\right|^{2}$; in case (B), aiming at investigating on the behavior of the resonator in the situation where the light is totally trapped in the cavity, we perform the analysis for $\left|\mathbf{t}_{21}\right|^{2}=0$ while varying $\left|\mathbf{t}_{12}\right|^{2}$. In both cases $a_{\mathrm{d}}{ }^{2}$ and $T_{\mathrm{RT}}$ are set to $0.1 \mathrm{~dB}$ and $100 \mathrm{~ns}$ respectively, while the phases of $\mathbf{r}_{12}$ and $\mathbf{r}_{21}$, both set to $\pi / 2$, were retrieved by deriving the 
equations for the wave interference at the coupling element of the simulating setup (see Eq. (B5) and (B7) in Appendix B). The results for case (A) are shown in Fig. 3a. We can see that $G_{\text {cav }}$ and $G_{\mathrm{R}}$ have roughly the same value in the reciprocal case, given that the internal loss is small. However, by decoupling $\left|\mathbf{t}_{12}\right|^{2}$ and $\left|\mathbf{t}_{21}\right|^{2}, G_{\text {cav }}$ exponentially grows with increasing degree of nonreciprocity, taking its maximum value at the highest degree of nonreciprocity, while $G_{\mathrm{R}}$ decreases. A rather different scenario occurs in the case (B) described in Fig. 3b, where both $G_{\text {cav }}$ and $G_{\mathrm{R}}$ vary linearly with $\left|\mathbf{t}_{12}\right|^{2}$. In this case, since $\left|\mathbf{t}_{21}\right|^{2}$ is set to $0, G_{\mathrm{R}}$ is a linear function of the reflection coefficient of the front mirror and no cavity resonant mode is coupled out, while the growth of $G_{\mathrm{cav}}$ is due only to the in-coupling energy rate $\rho_{\mathrm{in}}$, as predicted by Eq. (2) and Eq. (3) respectively. In fact, in the extreme case where also $\left|\mathbf{t}_{12}\right|^{2}=0$, then $G_{\text {cav }}=0$ and $G_{\mathrm{R}}$ has a finite value since the incoming power is totally reflected by the front mirror. The righthand side of the figure shows the spectral distribution over one FSR of the intra-cavity power enhancement and the normalized reflected power related to some values of Figs. $3 \mathrm{a}$ and $3 \mathrm{~b}$. As expected, the bandwidth of the intra-cavity spectrum, which is given by: $\Delta \omega=\left(4 / T_{\mathrm{RT}}\right)$ $\sin ^{-1}\left\{\left[1-\left|\mathbf{r}_{21}\right| a_{\mathrm{d}}^{-1 / 2}\right] /\left[2\left(\left|\mathbf{r}_{21}\right| a_{\mathrm{d}}{ }^{-1 / 2}\right)^{-1 / 2}\right]\right\}$, gets narrower with increasing degree of nonreciprocity since $\left|\mathbf{t}_{21}\right|^{2}$ (which is equal to $1-\left|\mathbf{r}_{21}\right|^{2}$ ) decreases. We also note that the zero out-coupling transmission $\left(\left|\mathbf{t}_{21}\right|^{2}=0\right.$, green- and yellow-dashed panel) leads to a reflection spectrum that is no longer dependent on the frequency, since it includes only the contribution of the power reflected by the front mirror. Particularly, in the specific case of fully coupled input power $\left(\left|\mathbf{t}_{12}\right|^{2}=1\right.$, i.e. - maximum degree of nonreciprocity), the reflected power is null, meaning that the light is completely trapped inside the resonator, and dissipated via the internal loss. Importantly, in both cases the numerical results are in a good agreement with equations (2), (3) and (4). Therefore, the decoupling of the in- and out-coupling energy rates in a resonant system, a consequence of the induced nonreciprocity, can dramatically affect the balance between the reflected and intra-cavity power and significantly improve the power enhancement provided by the resonator. 
(a)

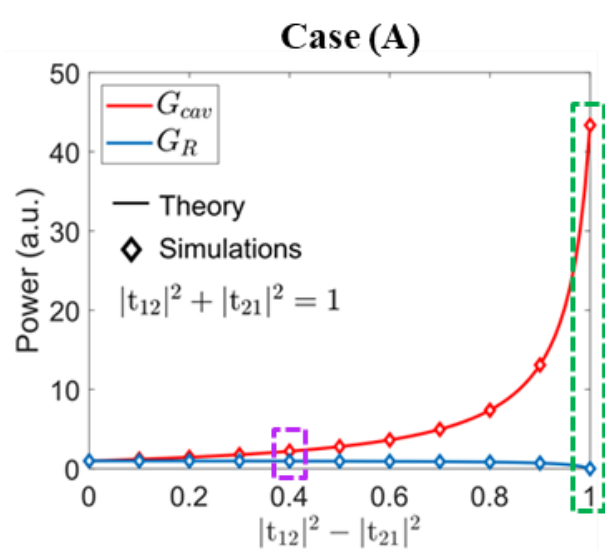

(b)

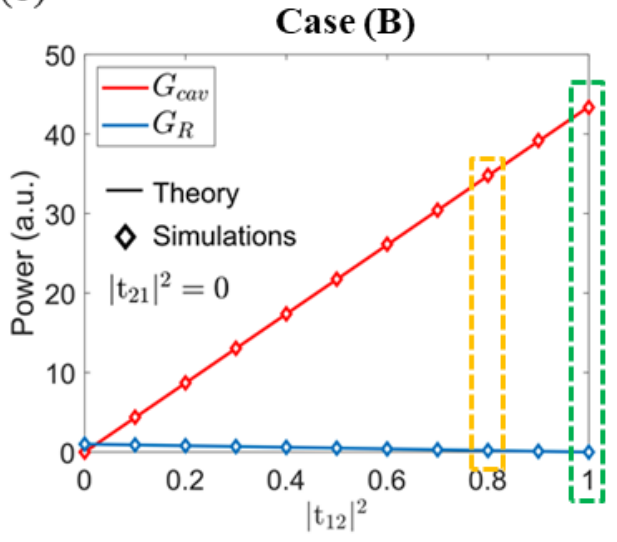

$|A(\omega)|^{2} /\left|A_{\text {in }}(\omega)\right|^{2} \quad\left|A_{R}(\omega)\right|^{2} /\left|A_{\text {in }}(\omega)\right|^{2}$
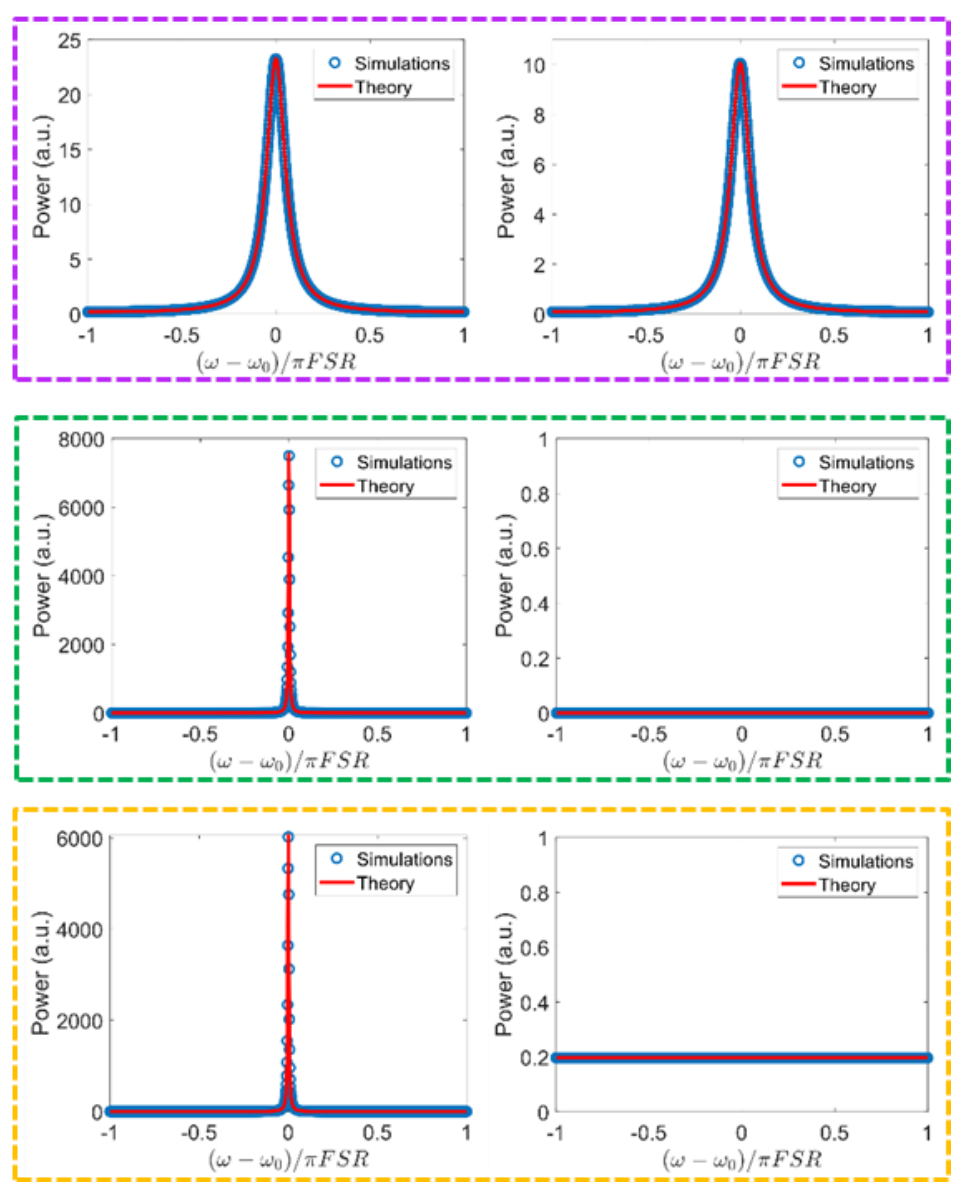

FIG. 3. Left-hand side: comparison of the values obtained from the simulations with those retrieved from equations (4) of the total power enhancement and reflected power, encased in one FSR and normalized to it. The graphs are related to the first (a) and the second (b) case of study and plotted as a function of the degree of nonreciprocity and the in-coupling transmittance respectively. Right-hand side: spectral distribution over one FSR of the reflected power and the intra-cavity power enhancement. The curves are related to a degree of nonreciprocity equal to 0.4 (violet-dashed panel) and 1 (greendashed panel), and to the case with $\left|\mathbf{t}_{21}\right|^{2}=0$ and $\left|\mathbf{t}_{12}\right|^{2}=0.8$ (yellow-dashed panel).

\section{EVALUATION OF THE TIME-BANDWIDTH PERFORMANCE}

Following the above analysis, we evaluate the TBP for different degrees of nonreciprocity. We recall that the resonator losses, which include both the radiative and non-radiative outcoupling energy rates, are quantified by the linewidth of the Lorentzian profile of the single resonant mode and not by the full-width-at-half-maximum (FWHM) of the Airy function characteristic of the resonator spectral response [59]. This is a rule that is valid regardless the strength of the coupling. Therefore, to properly derive the TBP, we use the TCMT to retrieve the Lorentzian mode profile associated to the loading and decay processes of the resonator. As explained in [43], analogously to the decay process of the energy stored in the resonator, which varies in time as $e^{-\left(\rho_{\mathrm{out}}+\rho_{0) t}\right.}$, and sets the bandwidth of the resonator to $\Delta \omega_{\mathrm{cav}}=\rho_{\text {out }}+\rho_{0}$, we 
can define a loading process, where the energy builds up in the resonator as $e^{+(\rho \text { in }+\rho 0) t}$ as the time is running backward [53]. Then, the loading rate $\left(\rho_{\text {in }}+\rho_{0}\right)$ represents the acceptance bandwidth of the resonator $\left(\Delta \omega_{\mathrm{acc}}=\rho_{\mathrm{in}}+\rho_{0}\right)$. This concept is illustrated in Fig. 4 , where both the loading and decay processes, and their associated bandwidths, are used to calculate the TBP of the system as $\Delta \omega_{\text {acd }} / \Delta \omega_{\text {cav }}[43]$.

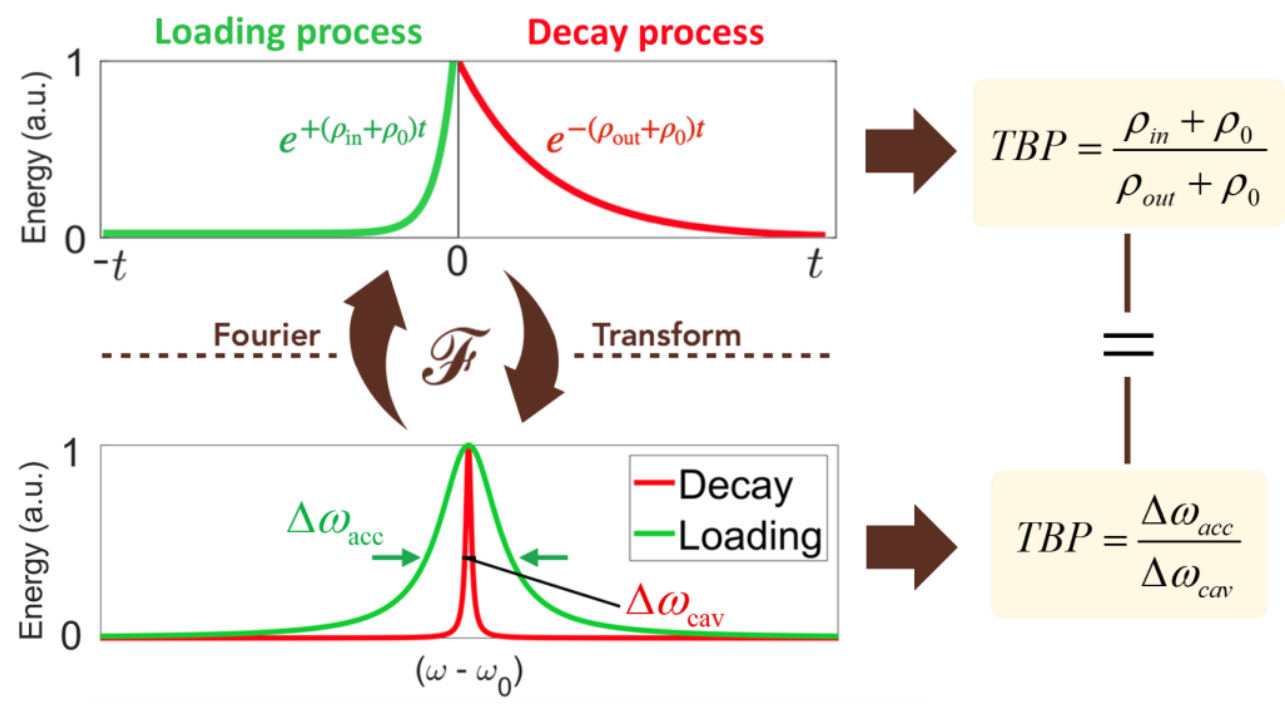

FIG. 4. Derivation of the TBP of a resonant system starting from its loading and decay processes. The TBP is given by the ratio between the acceptance and the cavity bandwidth which are the FWHM of the Lorentzian function associated, through the Fourier transform, to the loading and decay curve respectively.

We calculate the TBP of the resonator in the case of reciprocal and nonreciprocal coupling, and we plot the values in Fig. 5a as a function of $\left|\mathbf{t}_{12}\right|^{2}$. For the nonreciprocal case, we plot three curves corresponding to three values of the absorption loss $a_{\mathrm{d}}^{2}: 0$ (lossless), 0.1 and $0.5 \mathrm{~dB}$, while $\left|\mathbf{t}_{21}\right|^{2}$ is set to 0.1 . Owing to the decoupling of the in- and out-coupling energy rates, the TBP linearly increases with the increasing of $\left|\mathbf{t}_{12}\right|^{2}$ in case of nonreciprocal coupling, while it is always equal to 1 in case of reciprocal coupling. A similar scenario (for a time-invariant structure) was introduced in [42].

In Fig. $5 \mathrm{~b}$ the TBP of a resonator with $a_{\mathrm{d}}^{2}=0.1 \mathrm{~dB}$ is plotted for all the combinations of $\left|\mathbf{t}_{12}\right|^{2}$ and $\left|\mathbf{t}_{21}\right|^{2}$, with the red line indicating reciprocal coupling $\left(\left|\mathbf{t}_{12}\right|^{2}=\left|\mathbf{t}_{21}\right|^{2}\right)$. The highest TBP occurs at the maximum degree of nonreciprocity, while it becomes smaller than 1 when the degree of nonreciprocity is negative $\left(\left|\mathbf{t}_{12}\right|^{2}<\left|\mathbf{t}_{21}\right|^{2}\right)$. 
(a)

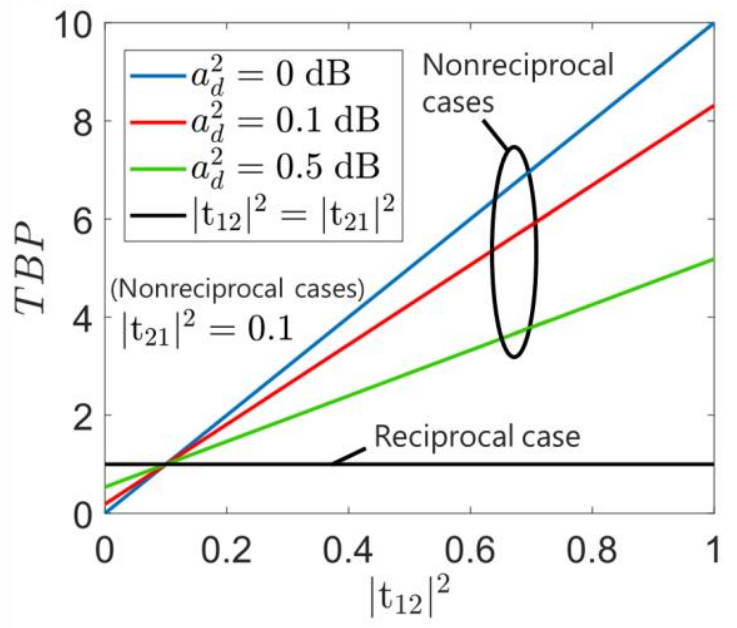

(b)

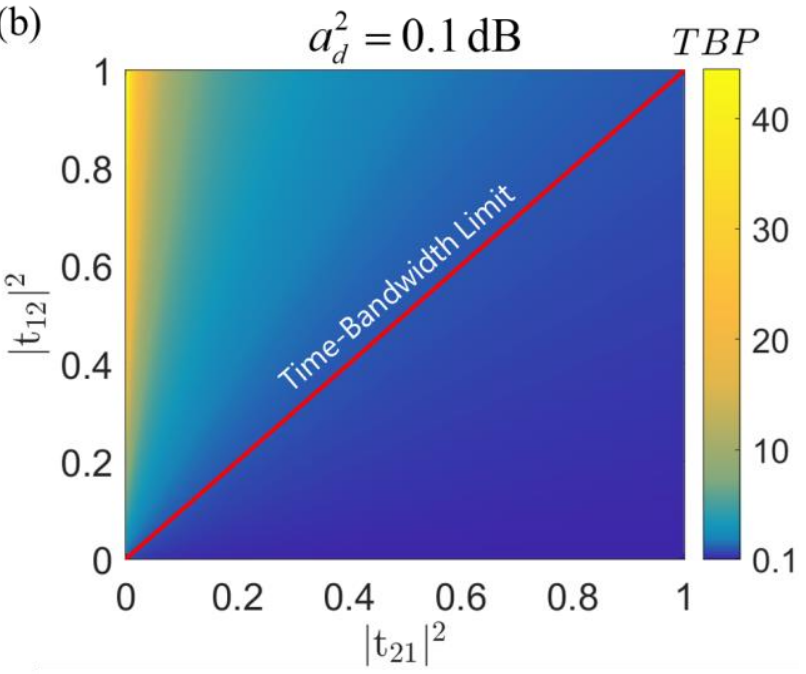

FIG. 5. (a) Comparison between the TBP of a reciprocal and a nonreciprocal resonator plotted as a function of the in-coupling transmittance. The curves of the nonreciprocal cases are relative to resonators with different absorption losses. (b) Graph of the TBP in color scale as a function of the inand out-coupling transmittance for a resonator with $0.1 \mathrm{~dB}$ of internal loss. The red line indicates the values relative to the reciprocal coupling (i.e. TBP $=1$ ), which corresponds to the Time-Bandwidth Limit.

It is interesting to note that the values of the TBP follow those of $G_{\text {cav }}$ in Fig. 3a showing a strict correlation between the TBP and the total power enhancement attained over one FSR. In fact, both show their peak at the maximum degree of nonreciprocity. However, while $G_{\text {cav }}$ is always null when $\left|\mathbf{t}_{12}\right|^{2}=0$, the TBP is greater than zero (and smaller than 1) because $\rho_{0} \neq 0$, and decreases with increasing $\left|\mathbf{t}_{21}\right|^{2}$ along the line $\left|\mathbf{t}_{12}\right|^{2}=0$, reaching its minimum at $\left|\mathbf{t}_{21}\right|^{2}=1$.

The benefit of the nonreciprocal coupling in a resonant system shows up more clearly by evaluating the total power enhancement in the nonreciprocal case with respect to the power enhancement achievable in the reciprocal case for the same amount of in-coupled power. This can be illustrated by plotting $G^{\mathrm{NR}}$ cav $/ G^{\mathrm{R}}$ cav , where $G^{\mathrm{NR}}$ cav is $G_{\text {cav }}$ for the nonreciprocal system calculated with $\rho_{\text {in }}>\rho_{\text {out }}$, and $G_{\text {cav }}^{\mathrm{R}}$ for the reciprocal one. The results, obtained from the simulations, are plotted in Fig. 6 as a function of the degree of nonreciprocity (with the same conditions of Case (A) in Fig. 3) and compared with the corresponding values of the TBP. Clearly, the ratio $G^{\mathrm{NR}}$ cav $/ G^{\mathrm{R}}$ cav increases exponentially with the degree of nonreciprocity, proving that by tailoring the decoupling of $\rho_{\text {in }}$ and $\rho_{\text {out }}$, the intra-cavity power can be enhanced much more than what could be done with a reciprocal resonator. We also note that the values of the TBP are in good agreement with those of $G_{\text {cav }}^{\mathrm{NR}} / G_{\text {cav }}^{\mathrm{R}}$, meaning that it can be used as a figure of merit to indicate the gain of total power enhancement due to nonreciprocal coupling, with respect to a reciprocal resonator, for an equal amount of in-coupled power. 


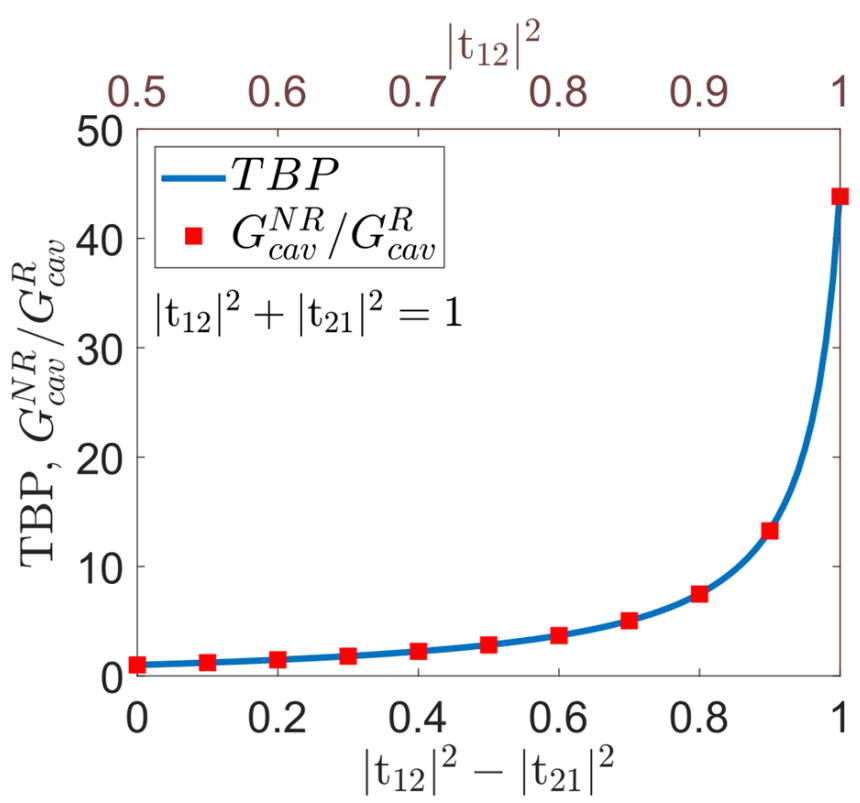

FIG. 6: Comparison between the values of the ratio $G^{\mathrm{NR}}$ cav $/ G^{\mathrm{R}}$ cav calculated using the values of $G_{\text {cav }}$ obtained from the simulations, and the TBP as a function of the degree of nonreciprocity of the system.

As a conclusive remark, let's consider the general definition of the TBP (TBP = $\left.\Delta \omega_{\mathrm{acc}} / \Delta \omega_{\mathrm{cav}}\right)[43]$. It can be rewritten as a ratio between the finesse related to $\Delta \omega_{\mathrm{cav}}$ and $\Delta \omega_{\mathrm{acc}}$, which we name cavity and acceptance Lorentzian finesse, $F_{\text {cav }}$ and $F_{\text {acc }}$ respectively:

$$
T B P=\frac{\Delta \omega_{\mathrm{acc}}}{\Delta \omega_{\mathrm{FSR}}} \frac{\Delta \omega_{\mathrm{FSR}}}{\Delta \omega_{\mathrm{cav}}}=\frac{\mathcal{F}_{\mathrm{cav}}}{\mathcal{F}_{\mathrm{acc}}}
$$

The physical meaning of this expression can be found by considering that the cavity finesse calculated using the Lorentzian linewidth represents the number of round-trips (times $2 \pi$ ) before the energy stored in the resonator decays to 1/e of its original value [37]. Applying this definition also to the loading process, we can say that the $F_{\text {acc }}$ is the number of round-trips the intra-cavity energy takes to reach its final value, starting from $1 / e$ of this value. Therefore, considering a certain amount of energy stored inside a resonator with $\rho_{\text {in }}>\rho_{\text {out }}$, a TBP $>1$ implies that the decay time $\tau_{\mathrm{D}}=1 / \rho_{\text {out }}+1 / \rho_{0}$ experienced by this energy is longer than that provided by a reciprocal resonator $(\mathrm{TBP}=1)$ by an amount equal to the ratio $F_{\text {cav }} / F_{\text {acc. }}$.

\section{CONCLUSION}

In conclusion, we investigated the implications of the nonreciprocal energy/power coupling on the TBP and the power balance of a generic resonant cavity. The results obtained by performing an analytical and numerical analysis of the frequency response of the modeled resonant system, show that the decoupling of the in- and out-coupling energy rates, as a consequence of the induced nonreciprocity, can significantly improve the power enhancement 
provided by the resonator compared to the reciprocal case. By evaluating the TBP of such a system, we show that it is greater (smaller) than 1 when $\rho_{\text {in }}>\rho_{\text {out }}\left(\rho_{\text {in }}<\rho_{\text {out }}\right)$ and takes its highest value at the maximum degree of nonreciprocity. We provide an interpretation of the $\mathrm{TBP}$ as a measure of the increased delay/storage time imparted to a light wave, with respect to what the bandwidth of the resonant structure would allow for the same amount of in-coupled power. This is valid for every finite value of the acceptance and cavity Lorentzian finesse and fits with the time-bandwidth limit $(\Delta \omega \cdot \tau=1)$ which, instead, simply links the cavity bandwidth to the photon life-time, only when the resonator is reciprocal. Moreover, by comparing the total power enhancement in the reciprocal and nonreciprocal case, we proved that the TBP is a figure of merit that characterizes the gain of total power enhancement attained over one FSR through nonreciprocal coupling compared to the reciprocal case considering the same amount of incoupled power. Understanding these fundamental relationships for general resonators will allow the development of novel optical devices, using nonreciprocity to further increase field enhancements and hence the efficiency of nonlinear optical interactions.

\section{APPENDIX A: DERIVATION OF EQUATIONS (2) AND (3)}

A general expression of the intra-cavity and reflected spectra of a Gires-Tournois resonator can be derived using the circulating field approach [46]:

$$
\begin{gathered}
A=\frac{\mathbf{t}_{12} \sqrt{a_{\mathrm{d}}}}{1-\left|\mathbf{r}_{21}\right| a_{\mathrm{d}} e^{-j \phi_{\mathrm{RT}}}} A_{\mathrm{in}} \\
A_{\mathrm{R}}=\left[\mathbf{r}_{12}+\frac{\mathbf{t}_{12} \mathbf{t}_{21} a_{\mathrm{d}} e^{-j\left(\phi_{\mathrm{RT}}-\phi_{21}^{\mathrm{r}}\right)}}{1-\left|\mathbf{t}_{21}\right| a_{\mathrm{d}} e^{-j \phi_{\mathrm{RT}}}}\right] A_{\mathrm{in}}
\end{gathered}
$$

where $a_{\mathrm{d}}=e^{-\alpha \mathrm{d} L_{\mathrm{RT}}}$ is the field inner circulation factor that accounts for the non-radiative loss of the resonator, $A_{\text {in }}$ is the amplitude of the input signal and $\phi_{\mathrm{RT}}$ is the total round-trip phase delay, which is given by the sum of the cavity round-trip phase delay $\phi_{\mathrm{d}}=\beta\left(2 L_{\mathrm{RT}}\right)$ and the phase of $\mathbf{r}_{21}\left(\phi_{21}\right)$. In these latter expressions, $L_{\mathrm{RT}}, \alpha_{\mathrm{d}}$ and $\beta$ are the cavity length, the intracavity power attenuation coefficient and the propagation constant, respectively. Given that $\phi_{\mathrm{RT}}$ $=2 m \pi+\Delta \phi_{\mathrm{RT}}$, where $\Delta \phi_{\mathrm{RT}}$ is the phase detune from resonance, the exponential term in Eq. (A1) and Eq. (A2) can be written as $e^{-j \Delta \omega T_{\mathrm{RT}}}$, where $\Delta \omega=\omega-\omega_{0}$, and $T_{\mathrm{RT}}=2 L_{\mathrm{RT}} / v_{\mathrm{g}}$ is the cavity round-trip time, with $v_{\mathrm{g}}$ the group velocity. Also, defining the intrinsic energy decay rate $\rho_{0}=\alpha_{\mathrm{d}} v_{\mathrm{g}}$, we can rewrite the inner circulation factor in the denominator of Eq. (A1) and Eq. (A2) as $a_{\mathrm{d}}=e^{-\left(\rho_{0} T_{\mathrm{RT}}\right) / 2}$. Using these relations and, recalling that the in- and out-coupling 
transmittances are related to their corresponding energy rates through $\rho_{\mathrm{in}}=\left|\mathbf{t}_{12}\right|^{2} / T_{\mathrm{RT}}$ and $\rho_{\mathrm{out}}=$ $\left|\mathbf{t}_{21}\right|^{2} / T_{\mathrm{RT}}$ [37], we can express the intra-cavity and reflected spectra as a function of $\rho_{\mathrm{in}}, \rho_{\text {out}}, \rho_{0}$ and $\Delta \omega$ :

$$
\begin{gathered}
A(\omega)=\frac{\sqrt{\rho_{\text {in }} T_{R T}} \sqrt{a_{\mathrm{d}}}}{1-\exp \left[\ln \left(\left|\mathbf{r}_{21}\right|\right)-\left(\rho_{0} / 2+j \Delta \omega\right) T_{\mathrm{RT}}\right]} A_{\text {in }}(\omega) \\
A_{\mathrm{R}}(\omega)=\left[\mathbf{r}_{12}+\frac{\sqrt{\rho_{\text {in }} \rho_{\text {out }}} T_{\mathrm{RT}} a_{\mathrm{d}} \exp \left[-j\left(\Delta \omega T_{\mathrm{RT}}-\phi_{21}^{\mathbf{r}}\right)\right]}{1-\exp \left[\ln \left(\left|\mathbf{r}_{21}\right|\right)-\left(\rho_{0} / 2+j \Delta \omega\right) T_{\mathrm{RT}}\right]}\right] A_{\text {in }}(\omega)
\end{gathered}
$$

\section{APPENDIX B: DESCRIPTION OF THE SETUP USED FOR THE SIMULATIONS}

The Gires-Tournois resonator can be experimentally implemented in the form of a figure9 cavity, as we have shown in [43], where the nonreciprocal front mirror is realized by the time-modulated Sagnac interferometer. We therefore build the numerical model of the GiresTournois resonator using the same layout used in [43]. To be able to explore the full nonreciprocal scenario consisting of an in-coupling transmittance spanning from 0 to 1 , we used a nonreciprocal coupler, instead of a standard directional coupler (see Fig. 7). In fact, as explained in [43], a standard directional coupler can lead, in presence of phase modulation, only to the maximum in-coupling transmittance $\left(\left|\mathbf{t}_{12}\right|^{2}=1\right)$. Therefore, as we will see, a nonreciprocity of the coupling coefficients of the coupler is required to unlock all the values of the in-coupling transmittance between 0 and 1 .
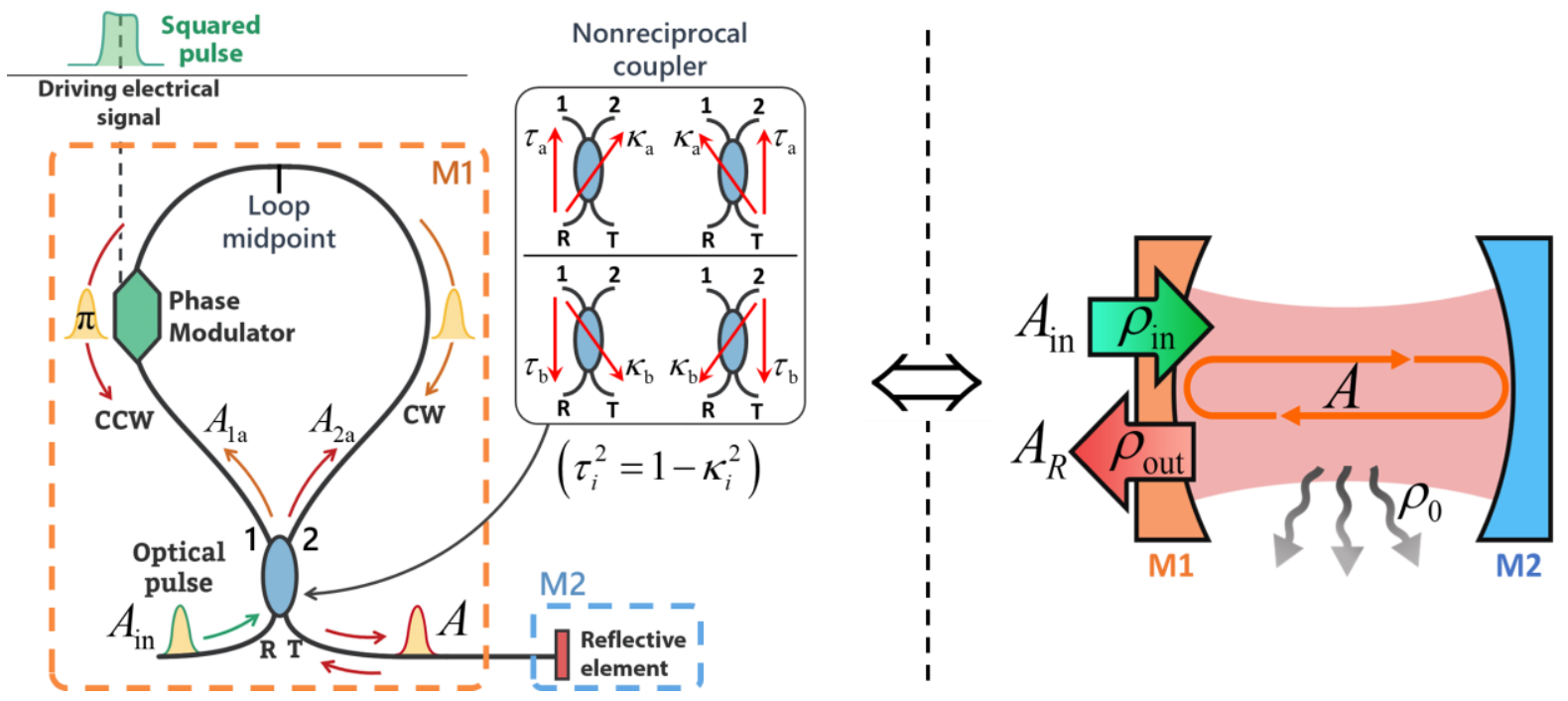

FIG. 7: Schematic representation of the setup used in VPIphotonics for the numerical simulations. The phase modulation in combination with the nonreciprocal coupler both integrated in a Sagnac interferometer ensures a total control of the transmission coefficient of the fiber loop, allowing to emulate the nonreciprocal front mirror of the Gires-Tournois resonator. 
In order to understand how the nonreciprocal coupling is simulated by the time-modulated Sagnac interferometer, we need to examine the equations that govern the wave interference at the coupler $[43,60]$.

Let us consider an optical pulse incident on the $\mathrm{R}$ port of the coupler, whose pulse duration is smaller than the cavity round trip time, $T_{\mathrm{RT}}$. Specifically, the pulse length is shorter than the distance of the phase modulator from the loop midpoint. This enables the impart of the phase modulation only to one of the two counter-propagating pulse.

The nonreciprocity of the coupler imposes that the cross and straight coupling coefficients depend on the direction of the light wave. In particular, $\kappa_{\mathrm{a}}$ and $\tau_{\mathrm{a}}$ are the cross and straight coupling coefficients respectively for the wave propagating from $\mathrm{R}$ or $\mathrm{T}$ port towards the inside the loop, while $\kappa_{\mathrm{b}}$ and $\tau_{\mathrm{b}}$ are the cross and straight coupling coefficients respectively for the wave going from inside the loop towards the R or T port. Explicitly, this means that for the left-hand schematic of Fig. 7, $\kappa_{\mathrm{a}}$ is the coupling efficient from $\mathrm{R}$ to 2 and $\mathrm{T}$ to $1, \tau_{\mathrm{a}}$ is for $\mathrm{R}$ to 1 and $\mathrm{T}$ to $2, \kappa_{\mathrm{b}}$ is for 2 to $\mathrm{R}$ and 1 to $\mathrm{T}$, while $\tau_{\mathrm{b}}$ is for 1 to $\mathrm{R}$ and 2 to $\mathrm{T}$. Since the input signal consists of a single pulse, it does not superimpose at the coupler with any other pulse (the same result would be obtained if the input signal was given by a pulse train with a period longer than the cavity RT time and not a multiple of it), the transfer characteristic of the coupler can be described by two distinct scattering matrices, one for each direction of propagation of the wave. We therefore can define $\mathbf{S}_{\mathrm{Ca}}$, the scattering matrix for the wave propagation towards the inside of the loop, and $\mathbf{S}_{\mathrm{Cb}}$ for the wave coming from within the loop:

$$
\mathbf{S}_{\mathrm{Ca}}=\left[\begin{array}{cc}
\tau_{\mathrm{a}} & -j \kappa_{\mathrm{a}} \\
-j \kappa_{\mathrm{a}} & \tau_{\mathrm{a}}
\end{array}\right] \quad \mathbf{S}_{\mathrm{Cb}}=\left[\begin{array}{cc}
\tau_{\mathrm{b}} & -j \kappa_{\mathrm{b}} \\
-j \kappa_{\mathrm{b}} & \tau_{\mathrm{b}}
\end{array}\right]
$$

A device with such characteristic is included in the VPIphotonics software library as a generic coupler in which it is possible to arbitrarily set the scattering parameters. However, the same behavior can be performed in practice by a time-variant tunable directional coupler made of a 4-port Mach-Zehnder interferometer [31].

Passing through the coupler the incoming pulse is split in two pulses whose complex amplitudes can be written as following:

$$
A_{1 \mathrm{a}}=\tau_{\mathrm{a}} A_{\text {in }} \quad A_{\text {2a }}=-j \kappa_{\mathrm{a}} A_{\text {in }}
$$

where $A_{\text {in }}$ is the original complex amplitude of the pulse. If there is no phase modulation the complex amplitudes of the two counter-propagating waves, the clockwise $(\mathrm{CW})$ and counterclockwise (CCW), are simply:

$$
A_{\mathrm{cw}}=\tau_{\mathrm{a}} A_{\text {in }} e^{-j \phi_{\mathrm{p}}} \quad A_{\mathrm{ccw}}=-j \kappa_{\mathrm{a}} A_{\mathrm{in}} e^{-j \phi_{\mathrm{p}}}
$$


where $\phi_{\mathrm{p}}$ is the phase delay acquired by the optical pulse through the fiber loop. Then, by denoting $A_{\mathrm{T}}$ and $A_{\mathrm{R}}$ the complex amplitudes of the transmitted and reflected portions of the pulse, respectively, the field transmission and reflection coefficients $\mathbf{t}_{0}$ and $\mathbf{r}_{0}$, respectively, are easily found:

$$
\begin{gathered}
A_{\mathrm{T}}=\tau_{\mathrm{b}} A_{\mathrm{cw}}-j \kappa_{\mathrm{b}} A_{\mathrm{ccw}} \Rightarrow \mathbf{t}_{0}=\frac{A_{\mathrm{T}}}{A_{\mathrm{in}}}=\left(\tau_{\mathrm{a}} \tau_{\mathrm{b}}-\kappa_{\mathrm{a}} \kappa_{\mathrm{b}}\right) e^{-j \phi_{\mathrm{p}}} \\
A_{\mathrm{R}}=-j \kappa_{\mathrm{b}} A_{\mathrm{cw}}+\tau_{\mathrm{b}} A_{\mathrm{ccw}} \Rightarrow \mathbf{r}_{0}=\frac{A_{\mathrm{R}}}{A_{\mathrm{in}}}=-j\left(\tau_{\mathrm{a}} \kappa_{\mathrm{b}}+\kappa_{\mathrm{a}} \tau_{\mathrm{b}}\right) e^{-j \phi_{\mathrm{p}}}
\end{gathered}
$$

where the subscript 0 indicates the absence of the phase modulation.

Conversely, if the phase modulator is electrically gated to shift by $\pi$ the phase of one of the two counter-propagating pulses only, say the CCW pulse, the complex amplitude $A_{\mathrm{ccw}}$ in Eq. (B3) becomes:

$$
A_{\text {ccw }}=-j \kappa_{\mathrm{a}} A_{\text {in }} e^{-j\left(\phi_{\mathrm{p}}+\pi\right)}=j \kappa_{\mathrm{a}} A_{\text {in }} e^{-j \phi_{\mathrm{p}}}
$$

and therefore, the complex transmission and reflection coefficients, $\mathbf{t}_{\pi}$ and $\mathbf{r}_{\pi}$, respectively, exhibited by the Sagnac interferometer will be:

$$
\mathbf{t}_{\pi}=\left(\tau_{\mathrm{a}} \tau_{\mathrm{b}}+\kappa_{\mathrm{a}} \kappa_{\mathrm{b}}\right) e^{-j \phi_{\mathrm{p}}} \quad \mathbf{r}_{\pi}=-j\left(\tau_{\mathrm{a}} \kappa_{\mathrm{b}}-\kappa_{\mathrm{a}} \tau_{\mathrm{b}}\right) e^{-j \phi_{\mathrm{p}}}
$$

where the subscript $\pi$ indicates the presence of the $\pi$ phase shift imparted by the phase modulator. In the above expressions, the value of $\phi_{\mathrm{p}}$ can be arbitrarily set to 0 without loss of generality.

When the electrical signal is applied at the phase modulator at a time $t_{1}$, and for a duration $t_{1} \leq t<t_{2}$, the Sagnac interferometer exhibits a transmission coefficient $\mathbf{t}\left(t_{1}\right)=\mathbf{t}_{\pi}$, while for the rest of the time $\left(t \geq t_{2}\right)$, the transmission coefficient is $\mathbf{t}\left(t_{2}\right)=\mathbf{t}_{0}$. Therefore, the localized timevarying phase modulation, in combination with the nonreciprocal coupler, allows to arbitrarily varying in time the power transmission coefficient ' $t$ ' of the Sagnac interferometer. In fact, we can obtain any value of $\mathbf{t}$ simply by acting on the electrical gating signal and by setting the proper parameters of the scattering matrices of the nonreciprocal couplers. The final result is two effective different coupling energy rates of the figure-9 resonator, one for $t_{1} \leq t<t_{2}$ and one for $t \geq t_{2}$ which are given respectively by:

$$
\rho\left(t_{1}\right)=\frac{\left|\mathbf{t}_{\pi}\right|^{2}}{T_{\mathrm{RT}}} \quad \rho\left(t_{2}\right)=\frac{\left|\mathbf{t}_{0}\right|^{2}}{T_{\mathrm{RT}}}
$$

If the incoming optical pulse is synchronized with the electric signal, it is coupled in the resonator through the Sagnac interferometer with a transmission coefficient $\mathbf{t}_{\pi}$, as it is depicted in Fig. 8a. However, while it resonates within the cavity, it is coupled out with a transmission 
coefficient $\mathbf{t}_{0}$ (Fig. 8b). Doing so, although the Sagnac interferometer exhibits a unique transmission coefficient at any given time, the incoming pulse experiences a transmission coefficient that is different from the one experienced by the intra-cavity pulse $\left(\mathbf{t}_{\pi} \neq \mathbf{t}_{0}\right)$. We can therefore identify $\mathbf{t}_{\pi}$ and $\mathbf{t}_{0}$ with the transmission coefficients $\mathbf{t}_{12}$ and $\mathbf{t}_{21}$, respectively, and $\rho\left(t_{1}\right)$ $\left(\rho\left(t_{2}\right)\right)$ with the in-coupling (out-coupling) energy rate $\rho_{\text {in }}\left(\rho_{\text {out }}\right)$ of the Gires-Tournois resonator. Analogously, we identify $\mathbf{r}_{\pi}$ and $\mathbf{r}_{0}$ with the reflection coefficient $\mathbf{r}_{12}$ and $\mathbf{r}_{21}$, respectively.
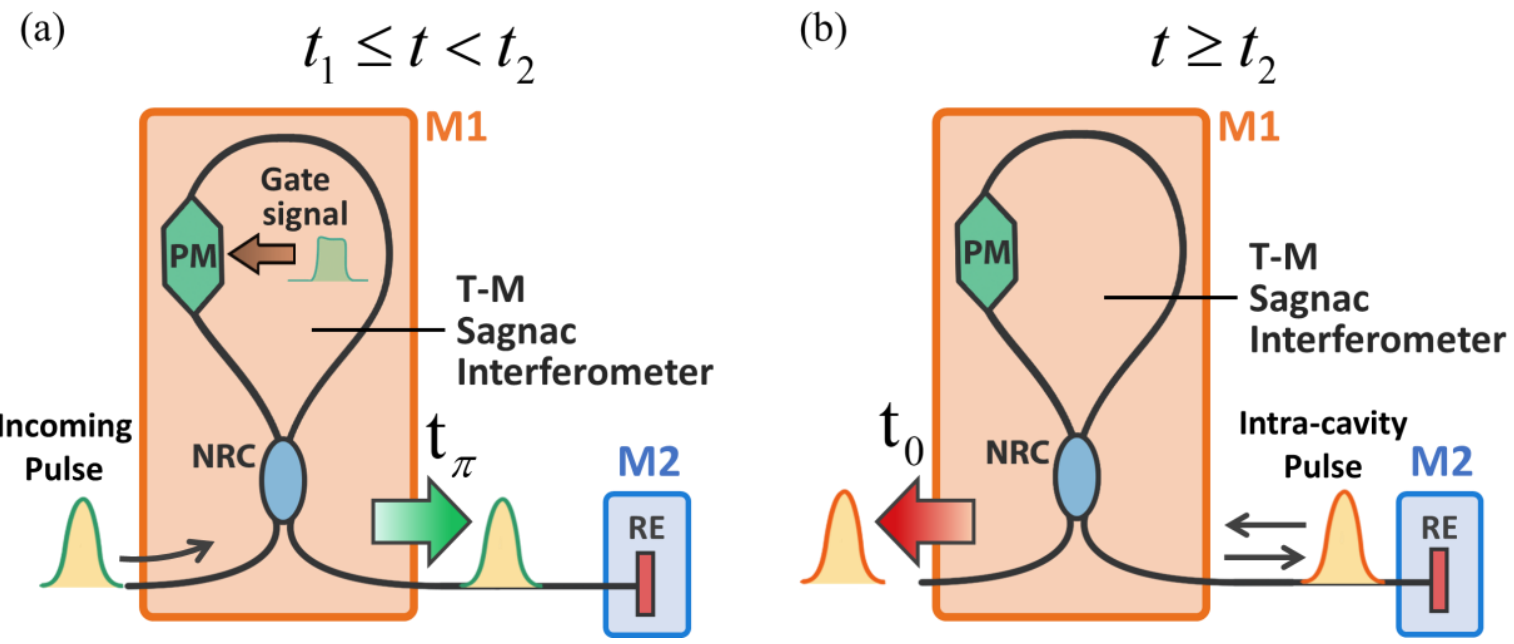

FIG. 8. Schematic representation of the Gires-Tournois resonator in the form of a figure- 9 cavity for (a) $t_{1} \leq t<t_{2}$ and for (b) $t \geq t_{2}$. PM: phase modulator; NRC: nonreciprocal coupler; RE: reflective element.

In such a system, the acceptance bandwidth corresponds to the bandwidth that the figure-9 resonator exhibits in the time window between $t_{1}$ and $t_{2}$, that is $\Delta \omega_{\text {acc }}=\rho\left(t_{1}\right)+\rho_{0}$, while the cavity bandwidth is the bandwidth that the system exhibits when there is no signal applied to the phase modulator (i.e. for $t \geq t_{2}$ ), which is given by $\Delta \omega_{\text {cav }}=\rho\left(t_{2}\right)+\rho 0$. Table 1 shows a summary of the parameters of the VPIphotonics simulative setup, based on the figure- 9 cavity implementation, and their corresponding parameters of the Gires-Tournois resonator model.

The choice of using a nonreciprocal coupler, instead of a standard directional coupler, can be ultimately clarified by calculating the expressions of $\mathbf{t}_{\pi}$ in case of a directional coupler with cross and straight coupling coefficients, $\kappa$ and $\tau$, respectively. Equation (B7) would then get the following form:

$$
\mathbf{t}_{\pi}=\left(\tau^{2}+\kappa^{2}\right) e^{-j \phi_{\mathrm{p}}}=1
$$

where we have assumed that the coupler is ideal, i.e. $\tau^{2}+\kappa^{2}=1$ and that $\phi_{\mathrm{p}}=0$. Therefore, in this case, it would not be possible to obtain an in-coupling transmittance different from 1 , thus 
limiting our parameter space. However, the nonreciprocal coupling is still ensured by the timevariant phase modulation, since $\mathbf{t}_{0}$ in this case would be given by:

$$
\mathbf{t}_{0}=\left(\tau^{2}-\kappa^{2}\right) e^{-j \phi_{\mathrm{p}}}
$$

In case of a 50/50 coupler Eq. (B10) gives: $\mathbf{t}_{0}=0$, which, together with Eq. (B9), represents the nonreciprocal coupling scenario explored experimentally in [43].

Table 1: Table summarizing the correspondences between the parameters of the VPIphotonics simulative figure-9-based setup and those of the Gires-Tournois resonator model.

\begin{tabular}{cc}
\hline VPIphotonics simulative setup & GT resonator model \\
\hline $\mathrm{t}_{\pi}=\left(\tau_{\mathrm{a}} \tau_{\mathrm{b}}+\kappa_{\mathrm{a}} \kappa_{\mathrm{b}}\right) e^{-j \phi_{\mathrm{p}}}$ & $\mathrm{t}_{12}$ \\
$\mathrm{t}_{0}=\left(\tau_{\mathrm{a}} \tau_{\mathrm{b}}-\kappa_{\mathrm{a}} \kappa_{\mathrm{b}}\right) e^{-j \phi_{\mathrm{p}}}$ & $\mathrm{t}_{21}$ \\
$\mathrm{r}_{\pi}=-j\left(\tau_{\mathrm{a}} \kappa_{\mathrm{b}}-\kappa_{\mathrm{a}} \tau_{\mathrm{b}}\right) e^{-j \phi_{\mathrm{p}}}$ & $\mathrm{r}_{12}$ \\
$\mathrm{r}_{0}=-j\left(\tau_{\mathrm{a}} \kappa_{\mathrm{b}}+\kappa_{\mathrm{a}} \tau_{\mathrm{b}}\right) e^{-j \phi_{\mathrm{p}}}$ & $\mathrm{r}_{21}$ \\
$\rho\left(t_{1}\right)=\left|\mathrm{t}_{\pi}\right|^{2} / T_{\mathrm{RT}}$ & $\rho_{\text {in }}$ \\
$\rho\left(t_{2}\right)=\left|\mathrm{t}_{0}\right|^{2} / T_{\mathrm{RT}}$ & $\rho_{\text {out }}$ \\
\hline
\end{tabular}

\section{Acknowledgments:}

KLT was supported by Hellenic Foundation for Research and Innovation (HFRI), General Secretariat for Research and Technology (GSRT) (No. 1819).

\section{References:}

[1] J. K. Jang, M. Erkintalo, J. Schroder, B. J. Eggleton, S. G. Murdoch, and S. Coen, "All-optical buffer based on temporal cavity solitons operating at $10 \mathrm{~Gb} / \mathrm{s}$," Opt. Lett. 41, 4526-4529 (2016).

[2] F. Leo, S. Coen, P. Kockaert, S. P. Gorza, P. Emplit, and M. Haelterman, "Temporal cavity solitons in one-dimensional Kerr media as bits in an all-optical buffer," Nat. Photonics 4, 471-476 (2010).

[3] M. D. Lukin and A. Imamoglu, "Nonlinear optics and quantum entanglement of ultraslow photons," Phys. Rev. Lett. 84, 1419-1422 (2000).

[4] H. Hu, D. Ji, X. Zeng, K. Liu, and Q. Gan, "Rainbow trapping in hyperbolic metamaterial waveguide," Sci. Rep. 3, 1249 (2013). 
[5] G. Demésy and S. John, "Solar energy trapping with modulated silicon nanowire photonic crystals," J. Appl. Phys 112, 074326 (2012).

[6] S. Eyderman, S. John, M. Hafez, S. S. Al-Ameer, T. S. Al-Harby, Y. Al-Hadeethi, and D. M. Bouwes, "Light-trapping optimization in wet-etched silicon photonic crystal solar cells," J. Appl. Phys 118, 023103 (2015).

[7] S. Eyderman and S. John, "Light-trapping and recycling for extraordinary power conversion in ultra-thin gallium-arsenide solar cells," Sci. Rep. 6, 28303 (2016).

[8] J. B. Khurgin, "Slow light in various media: a tutorial," Adv. Opt. Photonics 2, 287318 (2010).

[9] T. Baba, "Slow light in photonic crystals," Nat. Photonics 2, 465-473 (2008).

[10] M. González-Herráez, K.-Y. Song, and L. Thévenaz, "Optically controlled slow and fast light in optical fibers using stimulated Brillouin scattering," Appl. Phys. Lett 87, $081113(2005)$.

[11] S. A. Schulz, L. O'Faolain, D. M. Beggs, T. P. White, A. Melloni, and T. F. Krauss, "Dispersion engineered slow light in photonic crystals: a comparison," J. Opt. 12, 104004 (2010).

[12] L. Thévenaz, "Slow and fast light in optical fibres," Nat. Photonics 2, 474-481 (2008).

[13] T. F. Krauss, "Why do we need slow light?," Nat. Photonics 2, 448-450 (2008).

[14] S. John, "Why trap light?," Nat. Mater. 11, 997-999 (2012).

[15] K. L. Tsakmakidis, O. Hess, R. W. Boyd, and X. Zhang, "Ultraslow waves on the nanoscale," Science 358, eaan5196 (2017).

[16] C. W. Hsu, B. Zhen, J. Lee, S. L. Chua, S. G. Johnson, J. D. Joannopoulos, and M. Soljačić, "Observation of trapped light within the radiation continuum," Nature 499, 188-191 (2013).

[17] T. Tanabe, M. Notomi, H. Taniyama, and E. Kuramochi, "Dynamic release of trapped light from an ultrahigh-Q nanocavity via adiabatic frequency tuning," Phys. Rev. Lett. 102, 043907 (2009).

[18] J. Upham, Y. Fujita, Y. Kawamoto, Y. Tanaka, B. S. Song, T. Asano, and S. Noda, "The capture, hold and forward release of an optical pulse from a dynamic photonic crystal nanocavity," Opt. Express 21, 3809-3817 (2013).

[19] K. L. Tsakmakidis, A. D. Boardman, and O. Hess, "“Trapped rainbow" storage of light in metamaterials," Nature 450, 397-401 (2007).

[20] M. F. Yanik and S. Fan, "Stopping Light All Optically," Phys. Rev. Lett. 92, 083901 (2004). 
[21] M. F. Yanik and S. Fan, "Stopping and storing light coherently," Phys. Rev. A 71, 013803 (2005).

[22] H. J. S. Dorren, M. T. Hill, Y. Liu, N. Calabretta, A. Srivatsa, F. M. Huijskens, H. de Waardt, and G. D. Khoe, "Optical packet switching and buffering by using all-optical signal processing methods," J. Light. Technol. 21, 2-12 (2003).

[23] A. E. Willner, S. Khaleghi, M. R. Chitgarha, and O. F. Yilmaz, "All-optical signal processing," J. Light. Technol. 32, 660-680 (2014).

[24] Y. Okawachi, M. S. Bigelow, J. E. Sharping, Z. Zhu, A. Schweinsberg, D. J. Gauthier, R. W. Boyd, and A. L. Gaeta, "Tunable all-optical delays via brillouin slow light in an optical fiber," Phys. Rev. Lett. 94, 153902 (2005).

[25] Q. Gan, Z. Fu, Y. J. Ding, and F. J. Bartoli, "Ultrawide-bandwidth slow-light system based on thz plasmonic graded metallic grating structures," Phys. Rev. Lett. 100, 256803 (2008).

[26] J. Cardenas, M. A. Foster, N. Sherwood-Droz, C. B. Poitras, H. L. R. Lira, B. Yhang, A. L. Gaeta, J. B. Khurgin, P. Morton, and M. Lipson, "Wide-bandwidth continuously tunable optical delay line using silicon microring resonators," Opt. Express 18, 2652526534 (2010).

[27] F. Xia, L. Sekaric, and Y. Vlasov, "Ultracompact optical buffers on a silicon chip," Nat. Photonics 1, 65-71 (2007).

[28] L. Maleki, A. B. Matsko, A. A. Savchenkov, and V. S. Ilchenko, "Tunable delay line with interacting whispering-gallery-mode resonators," Opt. Lett. 29, 626-628 (2004).

[29] J. B. Khurgin and P. A. Morton, "Tunable wideband optical delay line based on balanced coupled resonator structures," Opt. Lett. 34, 2655-2657 (2009).

[30] W. Yoshiki, Y. Honda, T. Tetsumoto, K. Furusawa, N. Sekine, and T. Tanabe, "Alloptical tunable buffering with coupled ultra-high $\mathrm{Q}$ whispering gallery mode microcavities," Sci. Rep. 7, 8 (2017).

[31] W. V Sorin and R. S. Tucker, "Optical pulse storage, shaping, and wavelength conversion in resonators with controllable input coupling," J. Light. Technol. 27, 2587-2594 (2009).

[32] T. J. Kippenberg, R. Holzwarth, and S. A. Diddams, "Microresonator-based optical frequency combs," Science 332, 555-559 (2011).

[33] T. J. Kippenberg, A. L. Gaeta, M. Lipson, and M. L. Gorodetsky, "Dissipative Kerr solitons in optical microresonators," Science 361, eaan8083 (2018).

[34] R. S. Tucker, P.-C. Ku, and C. J. Chang-Hasnain, "Slow-Light Optical Buffers: 
Capabilities and Fundamental Limitations," J. Light. Technol. 23, 4046-4066 (2005).

[35] R. W. Boyd, D. J. Gauthier, A. L. Gaeta, and A. E. Willner, "Maximum time delay achievable on propagation through a slow-light medium," Phys. Rev. A 71, 023801 (2005).

[36] J. B. Khurgin, "Performance limits of delay lines based on optical amplifiers," Opt. Lett. 31, 948-950 (2006).

[37] V. Van, Optical Microring Resonators: Theory, Techniques, and Applications (CRC Press, 2017), pp. 35, 54, 75.

[38] R. S. Quimby, Photonics and Lasers: An Introduction (John Wiley \& Sons, 2006), p. 298.

[39] W. Demtröder, Laser Spectroscopy 1 (Springer, 2014), Vol. 1, p. 79.

[40] J. B. Khurgin, "Dispersion and loss limitation on the performance of optical delay lines based on coupled resonant structures," Opt. Lett. 32, 133-135 (2007).

[41] D. A. B. Miller, "Fundamental limit to linear one-dimensional slow light structures," Phys. Rev. Lett. 99, 203903 (2007).

[42] K. L. Tsakmakidis, L. Shen, S. A. Schulz, X. Zheng, J. Upham, X. Deng, H. Altug, A. F. Vakakis, and R. W. Boyd, "Breaking Lorentz reciprocity to overcome the timebandwidth limit in physics and engineering," Science 356, 1260-1264 (2017).

[43] I. Cardea, D. Grassani, S. J. Fabbri, J. Upham, R. W. Boyd, H. Altug, S. A. Schulz, K. L. Tsakmakidis, and C. S. Brès, "Arbitrarily high time bandwidth performance in a nonreciprocal optical resonator with broken time invariance," Sci. Rep. 10, 15752 (2020).

[44] D. L. Sounas and A. Alù, "Non-reciprocal photonics based on time modulation," Nat. Photonics 11, 774-783 (2017).

[45] F. Gires and P. Tournois, "Interferometre utilisable pour la compression d'impulsions lumineuses modulees en frequence," C. R. Acad. Sci. Paris 258, (1964).

[46] A. Yariv and P. Yeh, Photonics : Optical Electronics in Modern Communications, 6th ed. (Oxford University Press Inc, 2006), p. 160, 168.

[47] C. Caloz, A. Alù, S. Tretyakov, D. Sounas, K. Achouri, and Z. L. Deck-Léger, "Electromagnetic Nonreciprocity," Phys. Rev. Appl. 10, 047001 (2018).

[48] S. A. Mann, D. L. Sounas, and A. Alù, "Nonreciprocal Cavities and the TimeBandwidth Limit," Optica 6, 104-110 (2019).

[49] K. Tsakmakidis, Y. You, T. Stefański, and L. Shen, "Nonreciprocal cavities and the time-bandwidth limit: comment," Optica 7, 1097-1101 (2020). 
[50] A. Alu, S. Mann, and D. Sounas, "Nonreciprocal cavities and the time-bandwidth limit: reply," Optica 7, 1102-1107 (2020).

[51] V. Asadchy, M. S. Mirmoosa, A. Díaz-Rubio, S. Fan, and S. A. Tretyakov, "Tutorial on Electromagnetic Nonreciprocity and Its Origins," Proceedings of the IEEE 108, 1684-1727 (2020).

[52] A. Yariv, "Universal relations for coupling of optical power between microresonators and dielectric waveguides," Electron. Lett. 36, 321-322 (2000).

[53] H. A. Haus, Waves and Fields in Optoelectronics (Prentice-Hall, 1984), pp. 197, 228.

[54] S. Fan, W. Suh, and J. D. Joannopoulos, "Temporal coupled-mode theory for the Fano resonance in optical resonators," J. Opt. Soc. Am. A 20, 569-572 (2003).

[55] Z. Wang, S. Fan, and W. Suh, "Temporal coupled-mode theory and the presence of non-orthogonal modes in lossless multimode cavities," IEEE J. Quantum Electron. 40, 1511-1518 (2004).

[56] J. D. Joannopoulos, S. G. Johnson, J. N. Winn, and R. D. Meade, Photonic Crystals: Molding the Flow of Light, 2nd ed. (Princeton University Press, 2008), pp. 197-203.

[57] Z. Zhao, C. Guo, and S. Fan, "Connection of temporal coupled-mode-theory formalisms for a resonant optical system and its time-reversal conjugate," Phys. Rev. A 99, 33839 (2019).

[58] K. X. Wang, "Time-reversal symmetry in temporal coupled-mode theory and nonreciprocal device applications," Opt. Lett. 43, 5623-5626 (2018).

[59] N. Ismail, C. C. Kores, D. Geskus, and M. Pollnau, "Fabry-Pérot resonator: spectral line shapes, generic and related Airy distributions, linewidths, finesses, and performance at low or frequency-dependent reflectivity," Opt. Express 24, 1636616389 (2016).

[60] D. B. Mortimore, "Fiber Loop Reflectors," J. Light. Technol. 6, 1217-1224 (1988). 